\title{
Modeling of the Static Deflection of Positioning Table Supported by Multiple Linear Roller Bearings
}

[Hyeon-Il Oh, Jun-Ho Heo, Sun-Woong Kwon, Seong-Wook Hong*]

\begin{abstract}
Positioning tables supported by linear bearings are adopted for various machines such as material handling robots and machine tools. The accuracy of positioning tables is acknowledged to be essential for the machines employing the positioning tables. For instance, deflection of a positioning table for a machine tool during operation affects the machining accuracy for the machine tool. Thus, it is of great importance to accurately predict the deflections of the positioning tables in the design process. However, the deflections of positioning tables supported by multiple linear roller bearings (LRBs) are not easy to estimate because of the complicated characteristics of LRBs with regard to the system configuration and applied load. In this study, the static deflection of a large positioning table containing a number of LRBs was investigated. To this end, an improved simulation model for the deflection of a LRB subjected to vertical
\end{abstract}

load was employed. The static deflection model of a LRB took into account the contact deformation between the roller and rail, and between the roller and carriage. The elastic deformation of the carriage due to contact loads was also considered. The simulation model was further improved by rigorously comparing the simulation with the experiment for a LRB. Simulations were carried out to investigate the static deflection characteristics of a large positioning table containing multiple LRBs. Vertical loads were also considered to emulate the potential loading conditions in the simulation. The simulation results were presented and discussed. The presented method would be useful to predict the precision of a positioning table and then redesign or compensate the associated accuracy for the positioning table.

\section{Keywords-positioning table, linear roller bearing, static deflection}

\footnotetext{
Hyeon-Il Oh, Jun-Ho Heo, Sun-Woong Kwon, Seong-Wook Hong*

Kumoh National Institute of Technology

Republic of Korea

* Corresponding author
} 